\title{
Giant Mesenteric Cyst - Cause of Abdominal Distension Managed with Laparotomy-A Case Report
}

\author{
Nguyen $\mathrm{M}^{\star 1}$, Faul $\mathrm{P}^{2}$ and Naqvi $\mathrm{SA}^{1}$
}

${ }^{1}$ Department of Surgery, University Hospital Limerick, Ireland

${ }^{2}$ Department of Pathology, University Hospital Limerick, Ireland

${ }^{*}$ Corresponding author: Nguyen M, Department of Surgery, University Hospital Limerick, Ireland, E-mail: mnguyen@tcd.ie

\author{
Citation: Nguyen M, Faul P, Naqvi SA (2014) Giant Mesenteric Cyst - Cause of Abdominal Distension \\ Managed with Laparotomy-A Case Report. J Case Rep Stud 2(3): 303. doi: 10.15744/2348-9820.1.603
}

Received Date: March 07, 2014 Accepted Date: June 18, 2014 Published Date: June 20, 2014

\begin{abstract}
This case report describes the diagnosis and management of a large mesenteric cyst in a 55 year old lady who presented with abdominal distension \& with mass in the left upper quadrant. Mesenteric cysts are rare, benign, abdominal tumors to which $<1000$ cases have been reported in the literature. While $40 \%$ of cases are incidental findings found either through physical examination or imaging, they can cause non-specific abdominal symptoms including pain, altered bowel habits, nausea/vomiting or anorexia. Less commonly, $10 \%$ of cases can present with bowel obstruction, volvulus, torsion or shock. In general, the lack of characteristic clinical and radiological features presents as a diagnostic difficulty.

The mainstay in imaging is computerized tomography (CT). CT identifies and helps aid the decision to pursue a laparoscopic or open laparotomy approach, where complete surgical resection is the ultimate goal. In our patient a CT Abdomen \& Pelvis showed a large, loculated cystic mass measuring $30 \mathrm{~cm}$ in cranio-caudal length and $16 \mathrm{~cm}$ in the transverse and anterior/posterior diameter. While different approaches have been described in the literature to surgically resect such cysts, our approach was largely reflective of size and adherence to surrounding structures in this case. A laparotomy was performed using an upper mid-line $7 \mathrm{~cm}$ incision; $4500 \mathrm{cc}$ of fluid was aspirated from the cyst which was found to originate from the small bowel mesentery. A complete resection of the multi-loculated cystic sac was done that included the resection of the middle mesenteric vein. The post-operative period was uneventful. The patient was discharged on post-operative day 2. The Histopathology identified the mass as a multi-loculated peritoneal inclusion-type cyst.
\end{abstract}

\section{Introduction}

Mesenteric cysts are rare, abdominal tumours, they are benign growths with malignant transformation reported in 3\% of cases [14]. The incidence has been estimated to be 1 in 100,000 in the adult population and 1 in 20,000 in the pediatric population [1-3]. They often present in the first decade of life [5] with a 1:1 male to female preponderancW $[1,6]$.

They were first described during an autopsy in 1507 by an Italian anatomist, Benvenni. These lesions are often asymptomatic and found incidentally via physical examination or imaging, as in our case. $40 \%$ of cases are incidental findings with a palpable mass present in more than $50 \%$ of cases [3]. Less often they can present with non-specific abdominal symptoms including abdominal pain, anorexia, bowel habit changes, or nausea/vomiting. About $10 \%$ of cases can present as acute abdomen due to bowel obstruction, volvulus, and torsion or as shock secondary to bleeding or rupture $[2,4,7,8]$

Mesenteric cysts can occur in any part of the mesentery from the duodenum to the rectum [5]. They occur more often in the small intestine (66\%) than the large intestine (33\%) [5,6]. In the large bowel most arise from the right colon and ileum but rarely have been found in the mesentery of the descending colon, sigmoid colon and rectum $[3,9]$. The lining of mesenteric cysts are comprised of endothelial or mesothelial cells, usually cuboidal or columnar-type cells. They lack smooth muscle and lymphatic spaces (unlike cystic lymphangiomas) [4,6].

Imaging modalities such as ultrasound, computerized tomography (CT) and magnetic resonance imaging (MRI) are used in the diagnosis. However, only surgery can identify the site of origin [9]. Depending on size, laparoscopic approaches have yielded successful complete surgical resections [1,9] The first successful surgical resection was by Tillaux in 1880 and the first successful laparoscopic dissection was performed by Mackenzie in 1993 [2,5]. 


\section{Case}

Our case is a 55 year old lady who presented with a distended abdomen with abdominal mass. The patient initially presented to her General Practitioner (GP) for symptoms of utero-vaginal prolapse. On physical examination her GP palpated a large, smooth and mobile mass in the left upper quadrant. She was referred to the Surgical Assessment Unit at University Hospital Limerick for further investigations. She was asymptomatic (denied abdominal pain, irregular bowel habitus, no weight loss) apart from non-specific symptoms including abdominal fullness, decreased appetite and constipation. Her medical and surgical history was significant for hypothyroidism, utero-vaginal prolapse, and right inguinal hernia repair. Medications consisted of levothyroxine $150 \mathrm{mcg}$ BD.

Her laboratory investigations were normal (FBC, U\&E, coagulations, LFTs, CRP <3). Tumor marker CA-125 was also normal. An abdominal/pelvic ultrasound revealed a $21 \mathrm{~cm}$ multi-septated cystic mass arising from the left side of the abdomen. Findings were concerning for an ovarian neoplasm. In light of this suspicion she was reviewed by Gynaecology who ruled out an ovarian origin.

An Abdomen CT showed a large, loculated cystic mass measuring $30 \mathrm{~cm}$ in cranio-caudal length and $16 \mathrm{~cm}$ in the transverse and anterior-posterior diameter (Figure 1). There was intermittent association with the anterior border of the left kidney, pancreas and spleen; the cyst did not appear to arise from these structures. There was no involvement of ovarian tissue. Radiological impression was that of a large mesenteric cyst. Given her stable presentation, she was discharged and re-admitted for an elective removal of the cystic mass three weeks later.

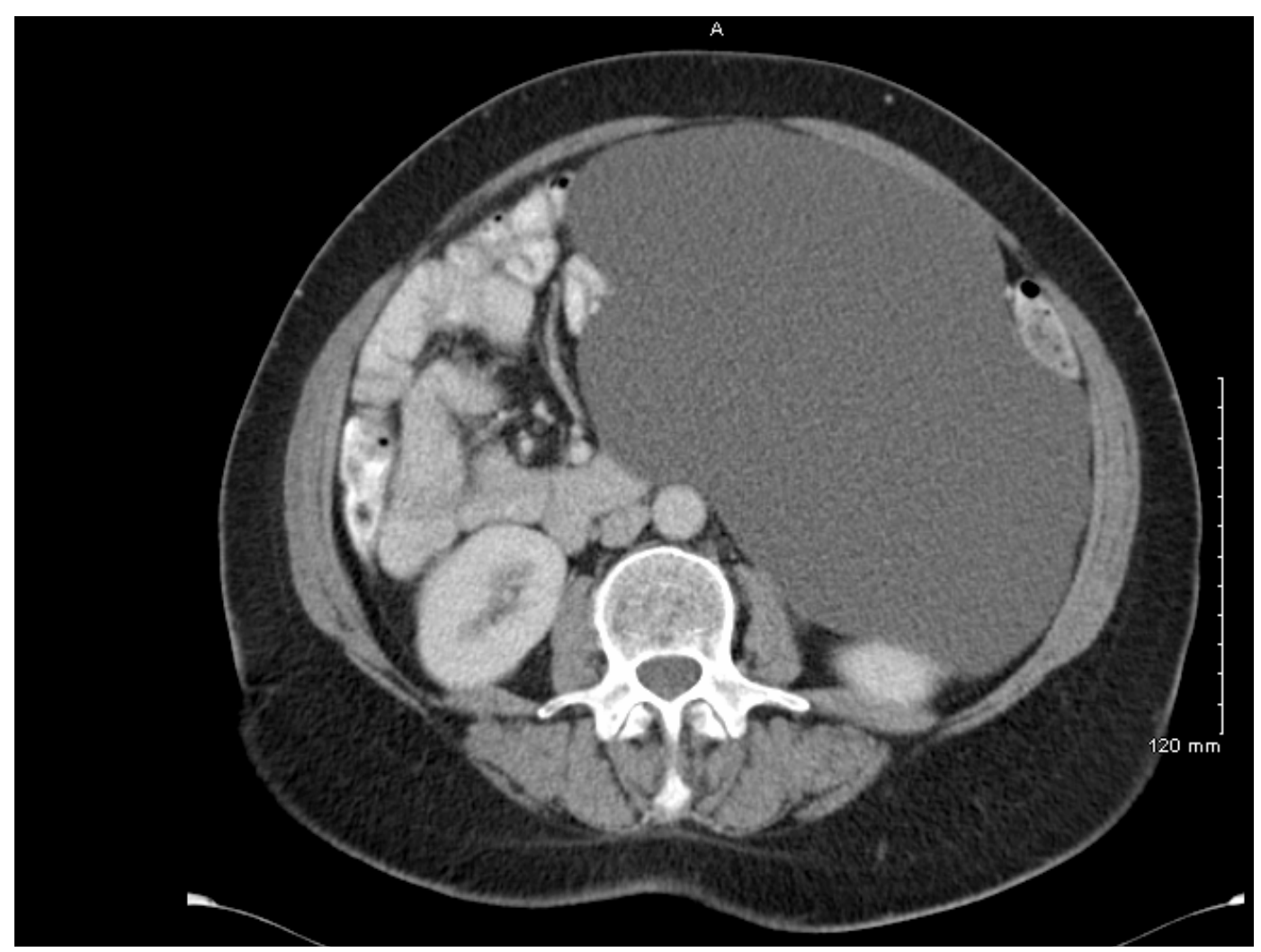

Figure 1: Abdominal CT, transverse view, displaying large cystic mass, measuring $30 \mathrm{~cm} \times 16 \mathrm{~cm} \mathrm{x} 16 \mathrm{~cm}$, accompanying majority of left abdomen

\section{Procedure}

The multi-loculated mesenteric cyst was excised electively under general anesthesia. A laparotomy was performed using an upper mid-line incision ( $7 \mathrm{~cm}$ wound) (Figure 2). Firstly, $4500 \mathrm{cc}$ of fluid was aspirated from the cyst then dissection followed.

The cyst originated from the small bowel mesentery. It was adherent within the small bowel to the left side of the sigmoid colon, and posterior aspect of the left kidney. On the right side it was attached to small intestinal mesentery, posteriorly with aorta and inferior vena cava. Superiorly, it was adherent to the third part of the duodenum. A large dissection was done including middle mesenteric vein. No intra-operative complications were recorded. The patient had a quick recovery and discharged shortly after the procedure (Figure 3). 
Grossly, it was $20 \mathrm{~cm} \times 15 \mathrm{~cm}$. The specimen was sent to cytopathology which revealed benign mesenteric cyst fluid containing macrophages with cyst contents. Histopathology identified a multi-loculated peritoneal inclusion-type cyst.

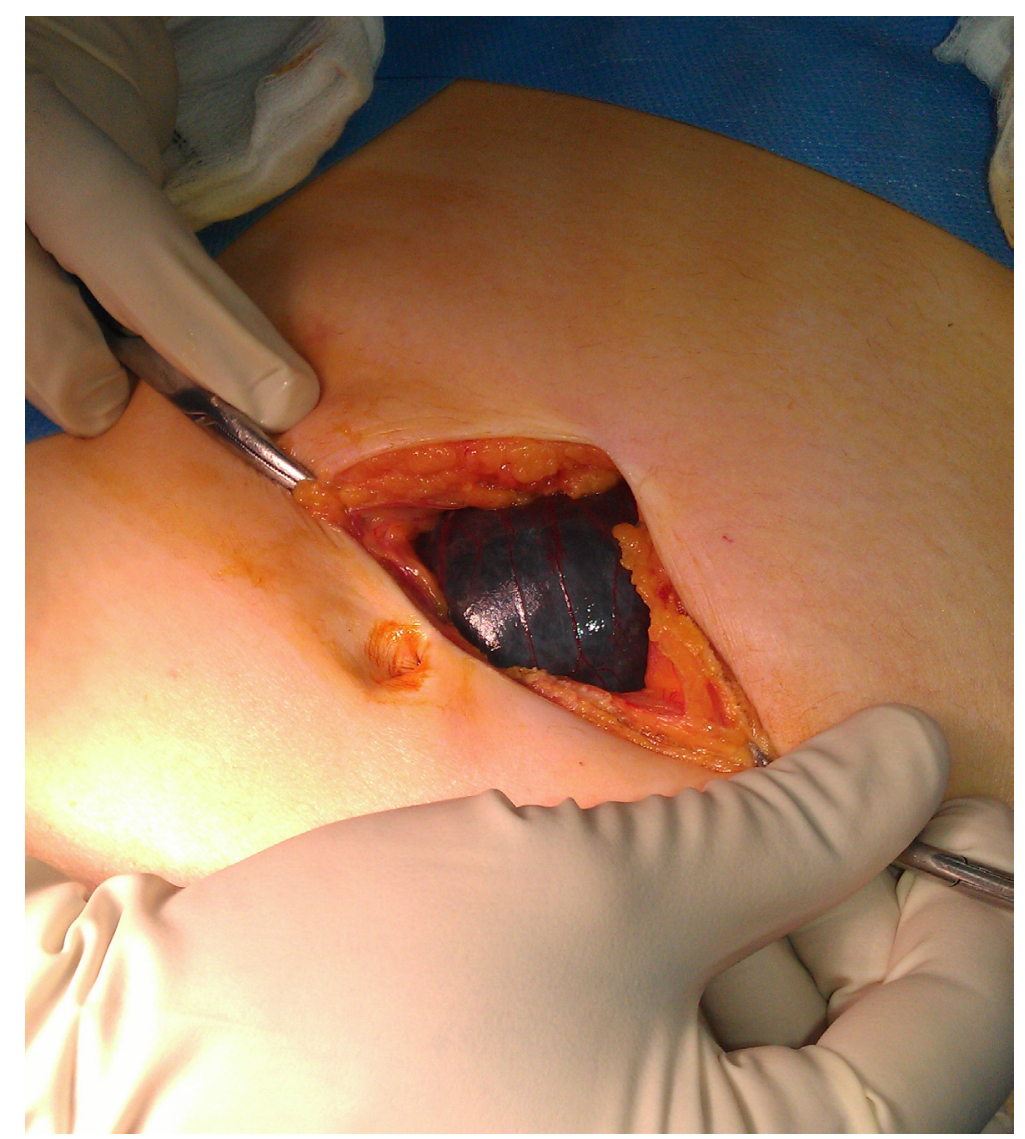

Figure 2: Midline laparotomy reveals a large mesenteric cyst just beneath the fascia.

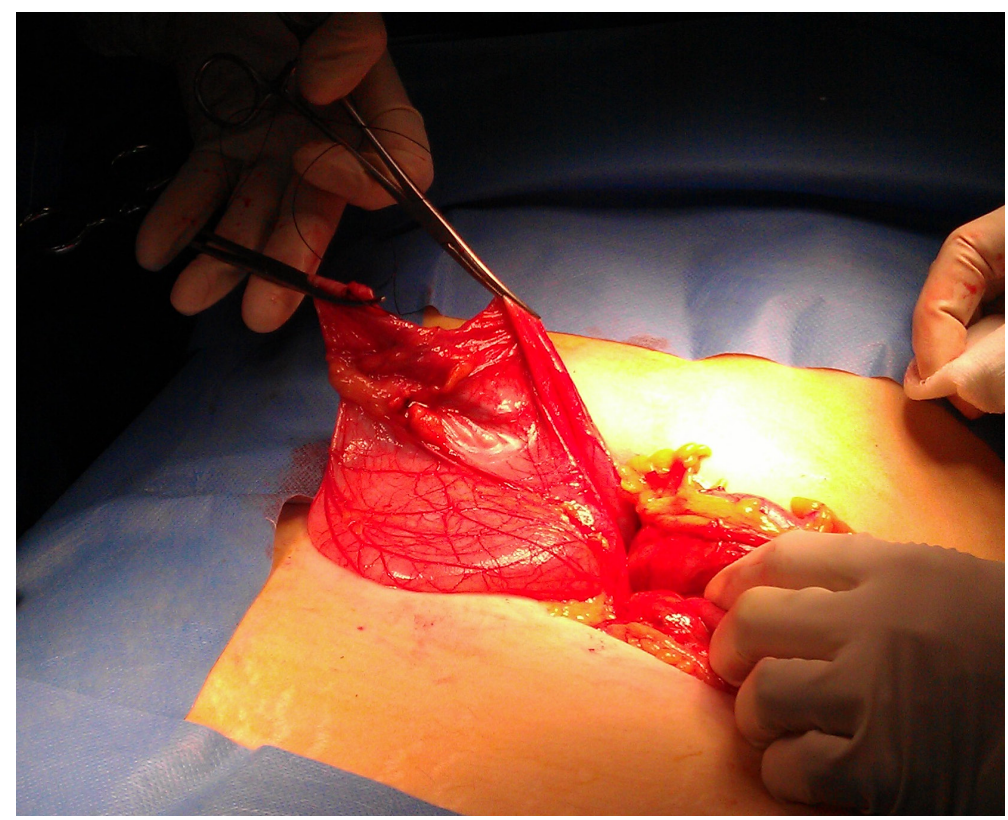

Figure 3: $4500 \mathrm{cc}$ was aspirated from the cyst. 


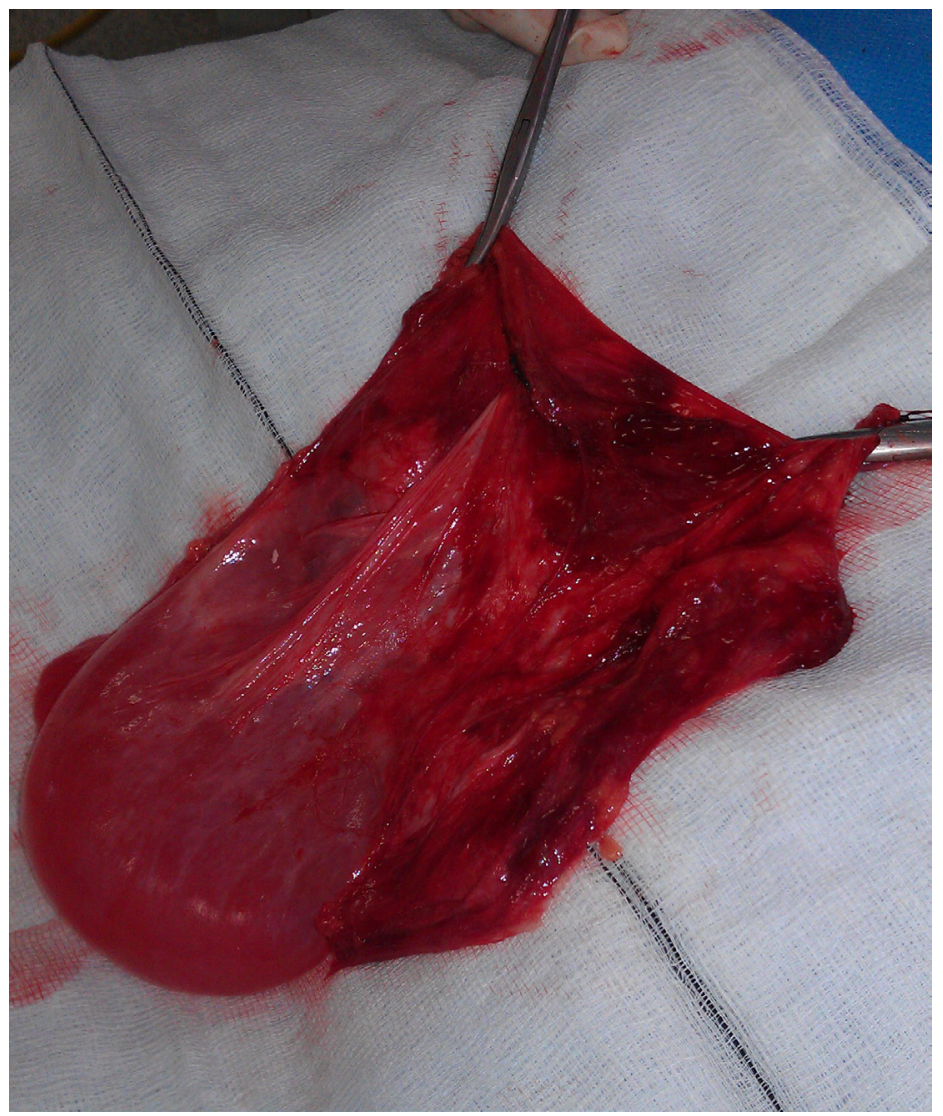

Figure 4: The cyst sac was excised.

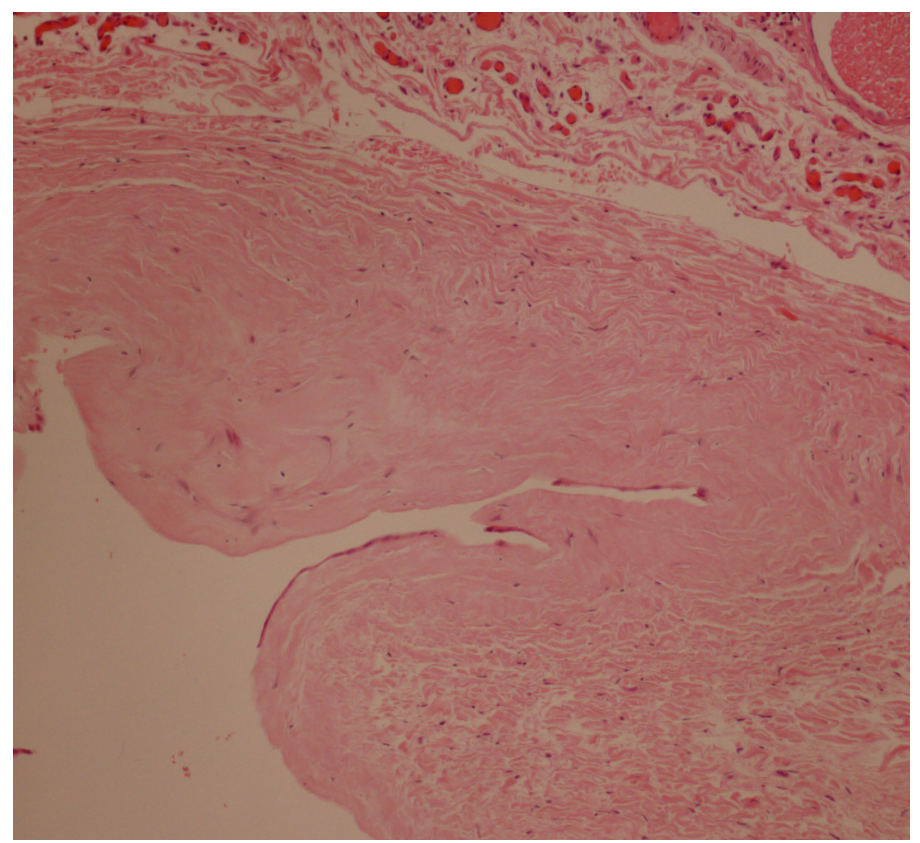

Figure 5: Haematoxylin-eosin stain. Histology under low power-multi-loculated peritoneal inclusion-type cyst. 


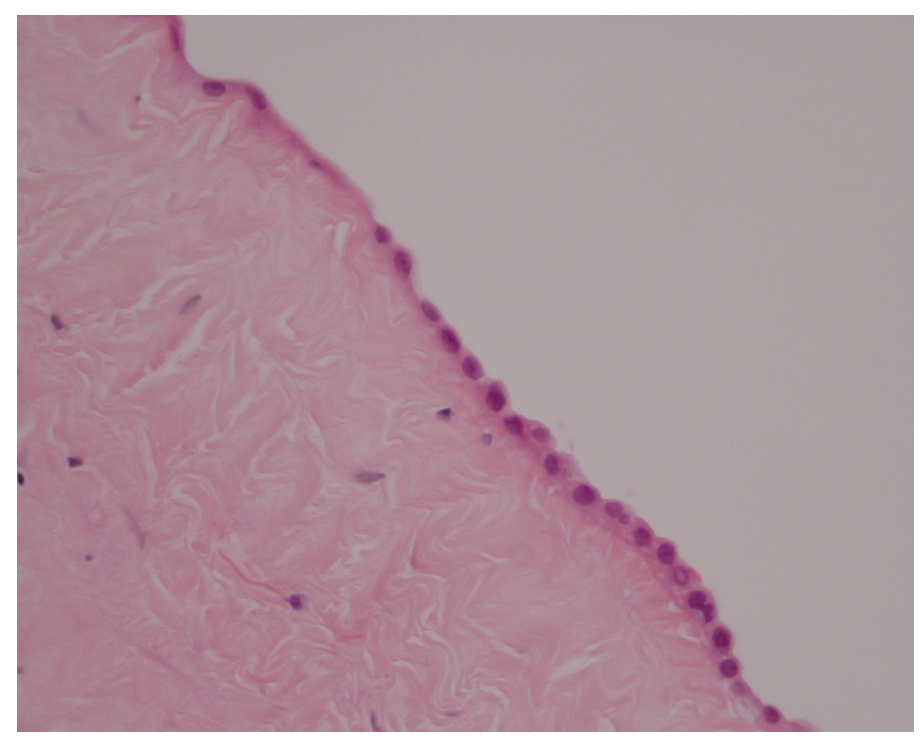

Figure 6: Haematoxylin-eosin stain. Histology under high power-multi-loculated peritoneal inclusion-type cyst.

\section{Discussion}

This case report demonstrates the presentation, diagnosis and surgical management of a large mesenteric cyst which was found incidentally with abdominal distension on physical examination. Mesenteric cysts are rare abdominal tumors that seldom present with overt abdominal symptoms. Abdominal pain is the most common symptom and an abdominal mass is found in more than $50 \%$ of cases [3]. Non-specific symptoms such as nausea, vomiting, weight loss, anorexia, weight loss and bloating may not be severe enough for patients to seek medical help. Their diagnosis is important however because they can present with acute symptoms such as hemorrhage, rupture, torsion, volvulus, or local compressive symptoms such as hydronephrosis, intestinal obstruction or lower limb oedema [2,3,7,8] (Figure 4).

On CT, mesenteric cysts can occur anywhere in the mesentery, from the duodenum to the rectum, and may extend into the retroperitoneum. They appear as cystic lesions that are further characterized by their wall thickness (thick or thin-walled) and their loculation (uni-locular or multi-locular) [10] (Figure 5, 6). Due to their cystic appearance, the differential diagnosis based on CT findings include pancreatic pseudocyst, ovarian cyst, lymphangioma, meconium pseudocyst, hydrometrocolpos, urachal cyst, hemangioma, endometriosis, loculated ascites (usually tuberculous), peritoneal inclusion cysts, cystic teratoma, cystic mesenteric panniculitis (sclerosing mesenteritis), hydatid cyst and urogenital cysts [10,11]. In general, the lack of characteristic clinical and radiological features presents as a diagnostic difficulty.

Localization of these cysts proves to be difficult pre-operatively. CT imaging is important in the management of these cysts as they can help aid the decision to pursue a laparoscopic or open laparotomy approach, when surgical resection is the goal. Other treatment options include drainage, marsupialization and enucleation which were previously thought to be the treatment of choice $[2,3,6]$. Bowel resection is required in a one third of adult and $50-60 \%$ of pediatric cases [6]. Partial excision is not indicated as there is a high recurrence rate with this modality [6]. Sometimes, complete resection and enucleation can not be achieved. In cases where the cyst is imbedded deep within the mesentery or when size is a factor, partial excision with marsupialization of the remainder of the cyst into the abdominal cavity (followed by schlerosis of the cyst lining) is a good option with low recurrence rates [6]. Average recurrence rates in a study with 162 adults and children were $6.1 \%$ in one study, and were more likely to occur in partial resections [6].

\section{Conclusion}

We have demonstrated that simple per operative controlled aspiration/drainage, followed by complete resection using a small abdominal wound via laparotomy approach is a successful surgical option of a very large mesenteric cyst. Surgical resection is considered the mainstay for therapy as recurrence may occur without complete resection $[2,3]$.

\section{References}

1. Memmo L, Belhaj A, Mehdi A (2013) Feasibility of laparoscopic resection of mesenteric cysts: two case reports. Acta Chir Belg 113: $43-6$.

2. Jain V, Demuro JP, Geller M, Selbs E, Romero C (2012) A case of laparoscopic mesenteric cyst excision. Case Rep Surg $2012: 594095$. 
3. Liew SC, Glenn DC, Storey DW (1994) Mesenteric cyst. Aust N Z J Surg 64: 741-4.

4. Ricketts RR (1998) Mesenteric and omental cysts. Pediatric Surgery 5th edn.

5. Wang JH, Lin JT, Hsu CW (2012) Laparoscopic excision of mesenteric duplication enteric cyst embedded in sigmoid mesocolon mimicking retroperitoneal neurogenic tumor in adults. Surg Laparosc Endosc Percutan Tech 22: e294-6.

6. Emedicine Medscape (2013) Mesenteric and omental cysts.

7. Blanco A, Sonntag C, Giese A (2013) Right lower quadrant abdominal pain--the usual suspects? Diagnosis and therapy of a symptomatic mesenteric cyst. Dtsch Med Wochenschr 138: 995-8.

8. Obaidah A, Mane SB, Dhende N, Acharya H, Thakur A (2012) Mesentric cyst- an unusual presentation as inguinal hernia. Indian J Surg 74: 184-5.

9. Bhandarwar AH, Tayade MB, Borisa AD, Kasat GV (2013) Laparoscopic excision of mesenteric cyst of sigmoid mesocolon. J Minim Access Surg 9: 37-9.

10. Radiopaedia.org (2014) Mesenteric cyst.

11. Pantanowitz L, Botero M (2001) Giant mesenteric cyst: A case report and review of the literature. The Internet Journal of Pathology 1: 2.

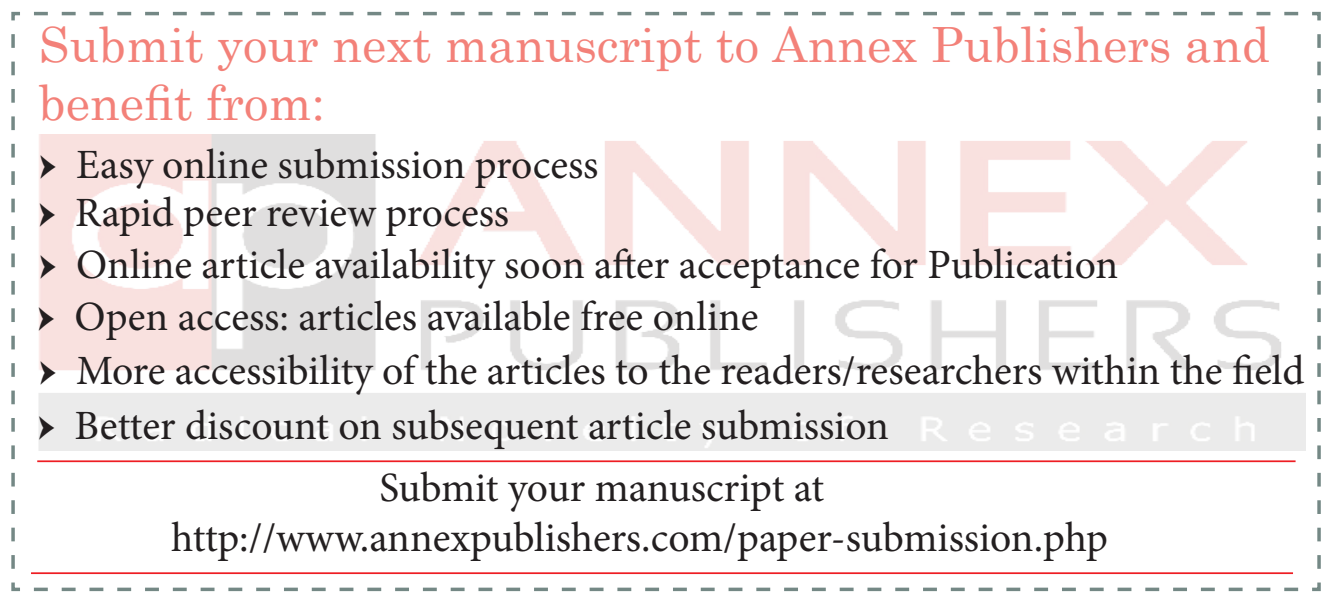

